\title{
H3K36me3 key to Polycomb-mediated gene silencing in lineage specification
}

\author{
Jumana AlHaj Abed and Richard S Jones \\ Department of Biological Sciences, Southern Methodist University, Dallas, Texas, USA \\ Richard S Jones: rjones@mail.smu.edu
}

\begin{abstract}
A newly uncovered activity of a family of Polycomb-group proteins provides insight into the mechanisms by which active genes become repressed during the transition from pluripotency to restricted cell fates as stem cells undergo lineage specification.
\end{abstract}

Polycomb group $(\mathrm{PcG})$ proteins are epigenetic regulators that maintain the transcriptional repression of numerous genes, many of which regulate developmental processes and/or cellcycle progression. Whereas the activities of many PcG proteins have largely been defined, the molecular contributions of Drosophila melanogaster Polycomblike (PCL) and its mammalian homologs, PHF1, PHF19 and MTF2, to PcG-mediated gene repression have remained elusive. Three studies in this issue ${ }^{1-3}$ reveal specific binding of the Tudor domains of PHF1, PHF19 and MTF2 to histone H3 trimethylated at lysine 36 (H3K36me3), a chromatin mark associated with transcriptionally active genes. These studies suggest a model by which PCL-family proteins may facilitate recruitment of PcG proteins to previously active genes, leading to de novo gene silencing.

Modification of chromatin structure, including the addition or removal of molecular tags on histones, has a central role in eukaryotic transcriptional regulation. In particular, nucleosomes near the transcription start site of active genes contain histone $\mathrm{H} 3$ trimethylated at lysine $4(\mathrm{H} 3 \mathrm{~K} 4 \mathrm{me} 3)$, whereas $\mathrm{H} 3 \mathrm{~K} 36 \mathrm{me} 3$ predominates in the transcribed bodies of genes. Conversely, $\mathrm{H} 3 \mathrm{~K} 9 \mathrm{me} 3$ and $\mathrm{H} 3 \mathrm{~K} 27 \mathrm{me} 3$ are associated with transcriptionally repressed genes.

Two primary PcG complexes, PRC1 and PRC2, are largely conserved in D. melanogaster and mammals. The core subunits of mammalian PRC2 are EZH2, SUZ12 and EED. The ARID domain-containing protein JARID2 is also a component of PRC2 in embryonic stem cells and is needed for recruitment of the complex to target genes ${ }^{4}$. Following recruitment of PRC2 to target genes through interaction with DNA-binding factors or noncoding RNAs, its catalytic subunit, EZH2, trimethylates H3K27. This serves as a molecular tag that facilitates recruitment of PRC1, although alternative modes of PRC1 recruitment are likely. Monoubiquitylation of histone $\mathrm{H} 2 \mathrm{~A}$ and/or chromatin compaction by $\mathrm{PRC} 1$ then inhibits

(C) 2012 Nature America, Inc. All rights reserved.

COMPETING FINANCIAL INTERESTS

The authors declare no competing financial interests. 
transcription of target genes ${ }^{5}$. Once PcG proteins have assumed control of a gene's expression, they may maintain its silence through an indefinite number of cell cycles. However, much less is known about the mechanisms involved in the initial establishment of PcG-mediated repression.

Variants of D. melanogaster and mammalian PRC2 have been purified that include PCL or one of three mammalian PCL homologs, PHF1, PHF19 or MTF2, in addition to core subunits. PCL-family proteins contain two PHD fingers, which interact with $D$. melanogaster $\mathrm{E}(\mathrm{Z})$ or mammalian $\mathrm{EZH} 2$, and an $\mathrm{N}$-terminal Tudor domain. However, a consensus for the mechanism(s) by which PCL-family proteins contribute to PRC2 activity has remained elusive. In vitro studies have suggested that addition of PCL or PHF1 to PRC2 enhances the complex's catalytic activity ${ }^{6-8}$. Other studies have suggested that PCL-family proteins are needed for recruitment of PRC2 to at least a subset of targets, including genes in embryonic stem cells that promote differentiation to more specialized cell types ${ }^{9,10}$. It is possible that PCL-family proteins may complement the activity of JARID2 in recruitment of PRC2 to target genes.

The pluripotency and proliferative capacity of embryonic stem cells require the expression of embryonic stem cell-specific transcription factors. Differentiation of embryonic stem cells into more specialized cell types requires concomitant activation of differentiationspecific genes and repression of embryonic stem cell-specific genes. PcG proteins are needed for this transition to fully repress embryonic stem cell-specific genes ${ }^{11}$. This represents an example of de novo repression of previously active genes and therefore must involve targeted recruitment of PRC2 and removal of the histone modifications associated with active genes, such as $\mathrm{H} 3 \mathrm{~K} 4 \mathrm{me} 3$ and $\mathrm{H} 3 \mathrm{~K} 36 \mathrm{me} 3$.

The new studies ${ }^{1-3}$ provide a model for how PCL-family proteins may play a central part in lineage transitions by recruiting PRC2 to previously active genes. Although the PHD fingers are known to mediate interaction with PRC2, the molecular activities of the PCL-family Tudor domain have remained undefined to date. Tudor domains, which consist of five antiparallel $\beta$-sheets that form a barrel-like fold, have since been identified in a wide variety of proteins and been shown to mediate protein interactions through recognition of methylated lysine or arginine residues ${ }^{12}$. In these new studies, the Tudor domains of PHF1, PHF19 and MTF2 are shown to specifically bind to H3K36me3, which requires conserved residues within an aromatic cage.

At first glance, this would seem counterintuitive, as H3K36me3 has been shown to inhibit the ability of PRC2 to methylate H3K27 (refs. 13,14). This conundrum is answered by PHF19-dependent recruitment of the histone demethylase NO66, which is capable of removing the inhibitory methyl groups from H3K36 (ref. 3) (Fig. 1a). A second H3K36me2 or H3K36me3 demethylase, KDM2b, also colocalizes with PHF19 and PRC2 at target genes in embryonic stem cells ${ }^{1}$, which suggests potential redundancy of these demethylases in assisting PcG-mediated repression. KDM2b is a component of an alternative form of PRC1, $\mathrm{BCOR}^{15}$. Therefore, it is not clear if KDM2b is directly recruited to PcG targets by PHF19PRC2 or whether it is present as a component of another PcG complex. 
PRC2 previously has been shown to directly interact with and recruit the H3K4me3 demethylase KDM5 $\mathrm{a}^{16}$. A second H3K4me3 demethylase, KDM5b, also has been shown to colocalize with PcG proteins at a subset of PcG target genes in embryonic stem cells, but it does not directly interact with PRC2 (ref. 17). Nucleosomes near the promoters of many PcG target genes in embryonic stem cells are marked by both H3K4me3 and H3K27me3. Such 'bivalent domains' may permit rapid activation of these genes as cells are induced to differentiate. The presence of KDM5a and/or KDM5b at these sites suggests that they may modulate $\mathrm{H} 3 \mathrm{~K} 4 \mathrm{me} 3$ rather than completely eliminate it.

When taken together with the current observations, an appealing model develops in which the combined activities of KDM5a (and possibly KDM5b), plus NO66 and/or KDM2b may remove both marks of transcriptionally active genes, H3K4me3 and H3K36me3, facilitating PcG-mediated silencing of previously active genes (Fig. 1b). The contribution of PHF19 in long-term maintenance of target-gene silencing is less clear. Like JARID2, PHF19 is also needed to maintain PRC2 at differentiation-specific genes in embryonic stem cells long after their initial silencing, at which point $\mathrm{H} 3 \mathrm{~K} 36 \mathrm{me} 3$ is no longer detectable. It is possible that maintenance of repression requires the continued presence of NO66 or KDM2b to inhibit the deposition of H3K36me3. Studies of D. melanogaster tissues revealed a surprisingly rapid turnover of PcG proteins at target genes ${ }^{18}$, which may necessitate constant vigilance against antagonistic chromatin modifiers. Alternatively, PCL-family proteins may maintain PRC2 at repressed genes by a mechanism independent of H3K36me3 binding and recruitment of H3K36me3 demethylases. One study ${ }^{3}$ finds that PHF19 may also be able to bind to H3K27me3, although with a lower affinity than it binds to H3K36me3, which could contribute to such maintenance. However, the findings of a second study show that the PHF19 Tudor domain specifically binds only to H3K36me3 (ref. 1).

Contrary to this model, it is suggested that H3K36me3 binding by PHF1 inhibits the catalytic activity of PRC2 (ref. 2). This raises an intriguing possibility that PRC2 activity may be modulated by inclusion of different PCL-family proteins. Although it is not yet tested, it may be that PHF1 does not recruit an H3K36me3 demethylase. However, it should be noted that previous studies have shown that PHF1 enhances PRC2 activity ${ }^{6,7}$. Further studies will be needed to resolve this apparent contradiction.

In addition to transcriptional repression, $\mathrm{PcG}$ proteins also have a role in response to DNA damage ${ }^{19}$. H3K36me3 quickly appears at sites of double-strand breaks (DSBs). Recruitment of PHF1 to DSBs previously has been shown to be mediated by its Tudor domain ${ }^{20}$. Musselman et al. ${ }^{2}$ confirm these findings and demonstrate that it is the PHF1 Tudor domain's ability to bind to $\mathrm{H} 3 \mathrm{~K} 36 \mathrm{me} 3$ that is responsible for this recruitment.

Unlike its mammalian counterparts, D. melanogaster PCL lacks two of the conserved residues in the aromatic cage of its Tudor domain and does not bind H3K36me3 (refs. 1,2), a finding consistent with previous observations that the PCL Tudor domain does not bind to any methylated histones ${ }^{21}$. This is somewhat curious, given the overall conservation of the PcG proteins' activities. However, a larger portion of PCL, that contains both the Tudor domain and first PHD finger, is shown to bind H3K36me3 and possibly also H3K4me3 and 
H3K9me3 (ref. 1). Thus, the role of PCL in recruiting PRC2 to target sites may be conserved but accomplished by a slightly different molecular mechanism.

Although H3K36me3 binding by PCL-family proteins is necessary for PRC2 recruitment and removal of the antagonistic modification by H3K36me3 demethylases, additional mechanisms must be responsible for specific targeting of the complex to only a small subset of previously active genes. Among the candidates are unidentified transcription factors, noncoding RNAs or localized chromatin modifications. Another unanswered question is whether PHF1 and MTF2, as well as D. melanogaster PCL, also recruit H3K36me3 demethylases. Should any of the mammalian PCL-family proteins lack this ability, this would raise the intriguing potential for modulation of PRC2 activity by substituting PCLfamily components of the complex.

\section{References}

1. Ballaré C, et al. Nat Struct Mol Biol. 2012; 19:1257-1265. [PubMed: 23104054]

2. Musselman A, et al. Nat Struct Mol Biol. 2012; 19:1266-1272. [PubMed: 23142980]

3. Brein G, et al. Nat Struct Mol Biol. 2012; 19:1273-1281. [PubMed: 23160351]

4. Landeira D, Fisher AG. Trends Cell Biol. 2011; 21:74-80. [PubMed: 21074441]

5. Simon JA, Kingston RE. Nat Rev Mol Cell Biol. 2009; 10:697-708. [PubMed: 19738629]

6. Cao R, et al. Mol Cell Biol. 2008; 28:1862-1872. [PubMed: 18086877]

7. Sarma K, et al. Mol Cell Biol. 2008; 28:2718-2731. [PubMed: 18285464]

8. Nekrasov M, et al. EMBO J. 2007; 26:4078-4088. [PubMed: 17762866]

9. Savla U, et al. Development. 2008; 135:813-817. [PubMed: 18216170]

10. Casanova M, et al. Development. 2011; 138:1471-1482. [PubMed: 21367819]

11. Pasini D, et al. Mol Cell Biol. 2007; 27:3769-3779. [PubMed: 17339329]

12. Pek JW, et al. Development. 2012; 139:2255-2266. [PubMed: 22669818]

13. Yuan W, et al. J Biol Chem. 2011; 286:7983-7989. [PubMed: 21239496]

14. Schmitges FW, et al. Mol Cell. 2011; 42:330-341. [PubMed: 21549310]

15. Sánchez C, et al. Mol Cell Proteomics. 2007; 6:820-834. [PubMed: 17296600]

16. Pasini D, et al. Genes Dev. 2008; 22:1345-1355. [PubMed: 18483221]

17. Schmitz SU, et al. EMBO J. 2011; 30:4586-4600. [PubMed: 22020125]

18. Ficz G, et al. Development. 2005; 132:3963-3976. [PubMed: 16079157]

19. Vissers JH, et al. J Cell Sci. 2012; 125:3939-3948. [PubMed: 23104738]

20. Hong Z, et al. Nucleic Acids Res. 2008; 36:2939-2947. [PubMed: 18385154]

21. Friberg A, et al. Protein Sci. 2010; 19:1906-1916. [PubMed: 20669242] 
a

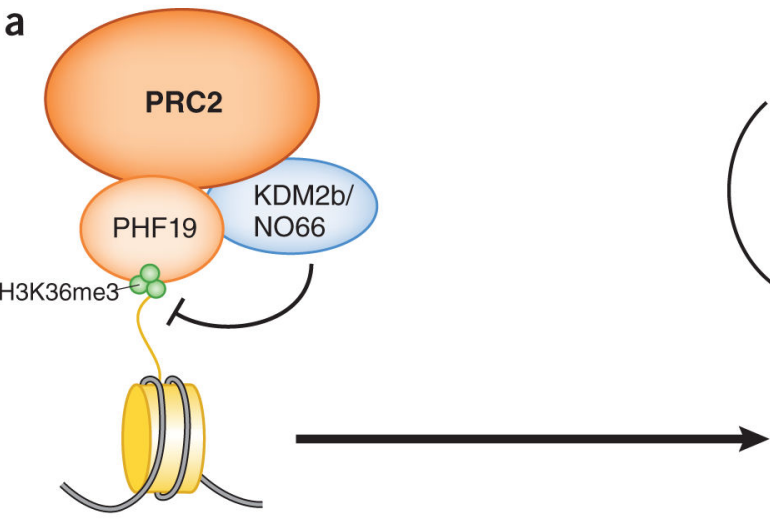

Gene on

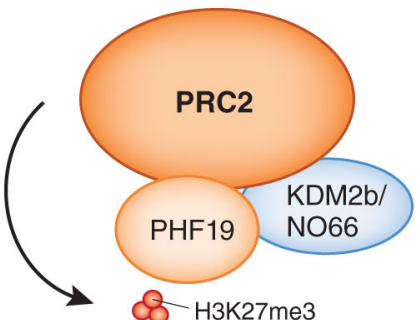

8 H $\mathrm{HK} 27 \mathrm{me} 3$

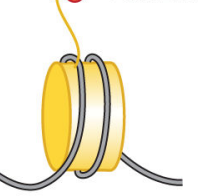

Gene off

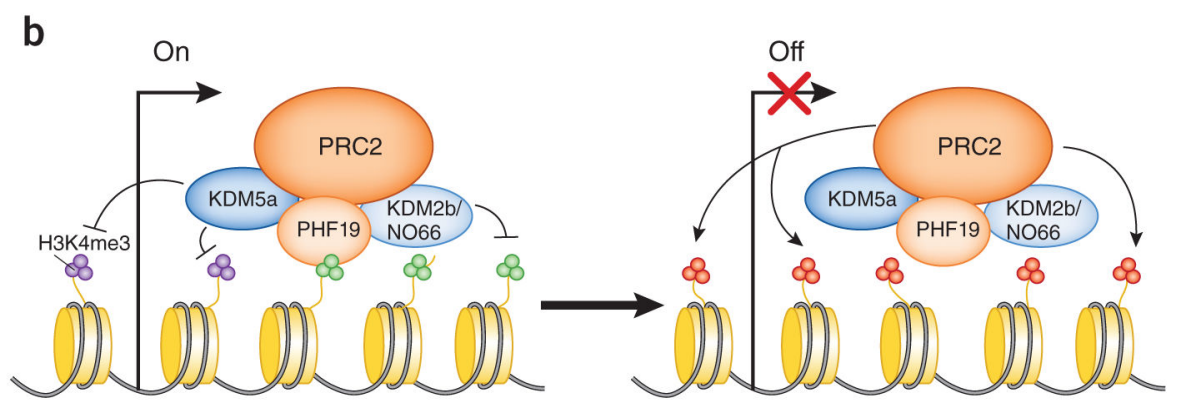

Figure 1.

H3K36me3 binding by PHF19 leads to recruitment of PRC2 and de novo gene silencing. (a) The Tudor domain of PHF19 binds H3K36me3 and recruits PRC2 and H3K36me3 demethylases NO66 and KDM2b (also known as Fbx110). H3K36me3 inhibits the catalytic activity of PRC2. Following demethylation of H3K36, PRC2 is able to trimethylate H3K27. (b) Recruitment of both $\mathrm{H} 3 \mathrm{~K} 4 \mathrm{me} 3$ demethylase KDM5a (also known as Rbp2 and Jarid1a) and H3K36me3 demethylases NO66 and/or KDM2b leads to removal of histone modifications near the promoter and downstream regions of active genes. This is followed by $\mathrm{H} 3 \mathrm{~K} 27$ trimethylation by PRC2 and establishment of transcriptional silencing. 